\title{
STUDY OF TRABECULAR BONE IN RATS USING IMAGE PROCESSING
}

\author{
D. Jiménez-Mendoza
}

Universidad Autónoma de Querétaro, Facultad de Ingeniería Cerro de las Campanas s/n, C.P. 76010, Querétaro, México jmd_pepe@hotmail.com

D. Vargas-Vázquez

Universidad Autónoma de Querétaro, Facultad de Ingeniería Cerro de las Campanas s/n, C.P. 76010, Querétaro, México damianvv@uaq.mx

\section{M.I. Hernández-Urbiola}

Centro de Física Aplicada y Tecnología Avanzada (UNAM) Boulevard Juriquilla No. 3001, Juriquilla, C.P. 76230, Querétaro, México angel22272@yahoo.com

\section{J.G. Ríos-Moreno}

Universidad Autónoma de Querétaro, Facultad de Ingeniería Cerro de las Campanas s/n, C.P. 76010, Querétaro, México. riosg@uaq.mx

\section{Trejo-Perea}

Universidad Autónoma de Querétaro, Facultad de Ingeniería Cerro de las Campanas s/n, C.P. 76010, Querétaro, México mtp@uaq.mx

\section{M.E. Rodríguez-García}

Centro de Física Aplicada y Tecnología Avanzada (UNAM) Boulevard Juriquilla No. 3001, Juriquilla, C.P. 76230, Querétaro, México marioga@fata.unam.mx

\footnotetext{
ABSTRACT

Osteoporosis is the most common osseous disease and it is considered a world problem. As the disease progresses, the bone structure diminish until it becomes very fragile and fractures risk up in region like femur and spine. The femoral neck and spine are spongy bone, which are formed by
} 
trabeculae in constant re-modeling regulated by osteoclast and osteoblast. When the balance between osteoclast and osteoblast is lost, removing bone process is faster than forming bone process, promoting the trabeculae thinning and eventually the osteoporosis. In order to count trabeculae, the experts traditionally use manual methods when they do not have automatic tools; this process introduces errors in the measurement. With the purpose of to count and to separate trabeculae, in this study a method is proposed that measure quantity, length and connections nodes in trabeculae regions, which are morphological characteristics indicators of the bone. The used images were obtained by an optical microscope starting with micro slides of bone. This approach provides an automatic tool of great utility in order to facility the process to the experts in medical area, and to avoid the cited errors in the manual count of trabeculae.

Keywords: Image Processing, Trabecula, Osteoporosis, Skeletonization

\section{INTRODUCTION}

Osteoporosis is considered a world problem: in Europe, U.S.A and Japan exist around of 75 million people with osteoporosis, and in Latin America this problem is growing up like other world parts ${ }^{1}$. Osteoporosis is a condition where bone mass gradually decreases until bone becomes very fragile and it breaks easily ${ }^{2}$. The bone quality is referring to the characteristics that determine the fracture risk ${ }^{3,4}$. The most common way to detect osteoporosis is by a densitometry study; through image processing algorithm of bone's part of interest as femur or spine, the osseous densitometer presents an approximation of the Bone Mineral Density (BMD) 5.

The femoral neck and spine are trabecular bones (spongy bone) which have a structure formed by trabeculae. When the width of the trabeculae is decreasing caused by osteoporosis, the connections among them can be lost and the bone becomes fragile ${ }^{6}$.

Bones with normal BMD have a trabecular structure uniformly distributed, it means, with quantity and width sufficient to have strong bones. In contrast, if the trabecular structure is thin with few connections nodes, the bone is fragile and it can break easily. For this reason, it is important the measurement of trabeculae which is possible too with image processing of 3D magnetic resonance in combination with the fuzzy distance transformation ${ }^{7}$; with this procedure it is calculated the approximation of distances among trabeculae and its thickness which indicates the trabecular distribution in bone. In order to follow the trabeculae shape, the 3D image is 
reduced by means of the skeletonization to determine the points where the trabeculae thickness is measured.

Other method used is through particle analysis and fractal index of trabecular regions ${ }^{8}$; with this technique, the trabeculae connection nodes are counted. The sensibility of the skeletonization methodology in the alterations of the trabecular bone pattern is analyzed in digital panoramic radiographies. The elements analyzed consist in five dry human mandibles. A skeleton was obtained from these $\mathrm{x}$-ray images, and these images were analyzed using: histogram, particle analysis, and fractal dimension.

$\mathrm{In}^{9}$, the lacunarity index has been employed in order to find differences among young trabecular bone, pre-menopausal trabecular bone, and osteoporotic trabecular bone in lumbar vertebra. The lacunarity function is approximated by a hyperbola model function which is applied to magnetic resonance images, the changes in those indexes can be found in the micro-structure density of the bone which indicates the beginning of osteoporosis; this analysis is performed by fractals properties in the micro-structure of bone.

The trabecular spaces can be calculated using ultrasound signals and an algorithm of inverse simplified filter ${ }^{10}$; using the correlation, the delay time, and the echo of signals, the trabecular spaces in cow's bone are calculated taking a phantom as a pattern. The trabecular space affects the ultrasound echo and the method analyzes the amplitude and phase of the dispersed echoes by the trabeculae; the obtained signal is processed with the inverse filter using the autocorrelation and spectral analysis of the signal.

The classification of trabecular regions from other different tissues can be analyzed using Fourier Transformation and Neural Networks ${ }^{11}$; images from bone biopsies are analyzed and separated in 3 groups (osteoporotic, osteopenic, and normal), the Fast Fourier Transformation is used to obtain the spectral frequency of the images. The spectrum frequencies of the images have remarkable differences among them, then the information of the spectral analysis is used to train a Neural Network; after this training process, the information is used as region classifier, separating the soft tissue from the trabeculae.

Another way to separate cells in the trabecular bone photography is by using an alternate sequential filter by reconstruction ${ }^{12}$. This work consists of the segmentation of biopsies where the image regions are differenced between them. Alternate sequential filter by reconstruction and a detection of homogeneous regions are used for separating these cells. Trabeculae extraction is performed by means of the morphological gradient, and they are labeled with a very low gray level. Because the fatty weave have a high 
gray level, a threshold operation is used to segment it. The non-labeled regions as trabeculae or woven fatty correspond to the hematopoietic weave. In the final stage, a study of the percentage of the different labeled region is performed considering the total of pixels in the image in order to estimate the trabecular region percentage.

Another alternative to measure trabeculae is presented by Blain et al. ${ }^{13}$; in this study the trabecular bone image is divided in four parts in order to count trabeculae; the trabecular distribution and a measurement of the cortical bone thickness of the samples were obtained by an automatic software analyzer (Bone-Explora Nova $\odot$ ). This system is specially designed to help the biologist explore and analyze bone slides according to international bone histology standards. The computer program delivers the trabecular distribution by means of fractal index using a skeleton of the region of interest (ROI).

In this work, the goal is developing an algorithm based on image processing in order to obtain morphologic measurements like trabeculae quantity, connection nodes, longitude, and trabeculae distribution in micro-slides of femoral neck of Wistar rats obtained by an optical microscope, in a similar way than some of the techniques described. The proposed methodology offers a tool to diminish execution times and errors in the manual counting process by experts. Another advantage not included in other methods is added: the measurement of quantity and length of the trabeculae; in addition, the quantity of connections nodes is performed in the same process.

\section{MATERIALS AND METHODS}

The femur's samples were obtained from Wistar rats; the specimens were maids with high quality standard in the bioterium of the Universidad Autonoma de Mexico (UNAM). The trabeculae micro-slides were delivered by Facultad de Medicina of Universidad Autonoma de Queretaro. The segmentation of trabecular bone is made in two parts, the first part is the camera calibration of the optic microscope, and the second one is the image processing that is described in following paragraphs.

\subsection{Camera and Microscope Setting}

An Optic microscope from Leica Microsystems $\bigcirc$ was used in this study, and it has a CMOS camera coupled to the system by USB connection. The camera was calibrated with a magnification of $12 x$ in a range from 4-40x. A calibration pattern and the software provided by the maker were used in order to calibrate the system; the lineal pixel measure was $0.0086 \mathrm{~mm}$. The light of the microscope was setting in the value of 8 in a 
scale from 1-10 levels. A black screen was used in the microscope in order to keep good brightness and to prevent differences among images. The photographs were taken with the Leica Application Suite v1.6@ software with $1024 \times 768$ pixels of resolution, and they were stored in BMP format; the selected resolution allows analyzing trabeculae details in an optimal way.

\subsection{Image Processing}

The color images obtained with the Leica software are stored in RGB (Red, Green, Blue) color space; therefore, in order to separate trabecular tissue from background, this color space was not the better option. Others color spaces were tested, and finally, the HSL color space ${ }^{14}$ was selected; this model is a representation in cylindrical coordinates of points in a RGB model, and perceptually more relevant than Cartesian representation. After of the conversion process, the Hue $(\mathrm{H})$, Saturation (S) and Lightness (L) components were analyzed. The image associated with the Lightness component (Equation 1) was the selected option to work, because this image shows notable differences between trabeculae tissue and the background. In order to reduce noise in the images, the median filter ${ }^{15}$ was applied in the Lightness image; this filter considers the median value of the pixels in the neighborhood of a $3 \times 3$ mask. In order to improve the contrast between background and trabeculae, the equalization of the histogram was applied (Figure 1). A region of interest (ROI) with a area of $2.947190 \mathrm{~mm} 2$ (200x200pixels) was chosen for analysis (Figure 2).

$$
L=\frac{\operatorname{Max}(R, G, B)+\operatorname{Min}(R, G, B)}{2}=\frac{\operatorname{Max}+\operatorname{Min}}{2}
$$

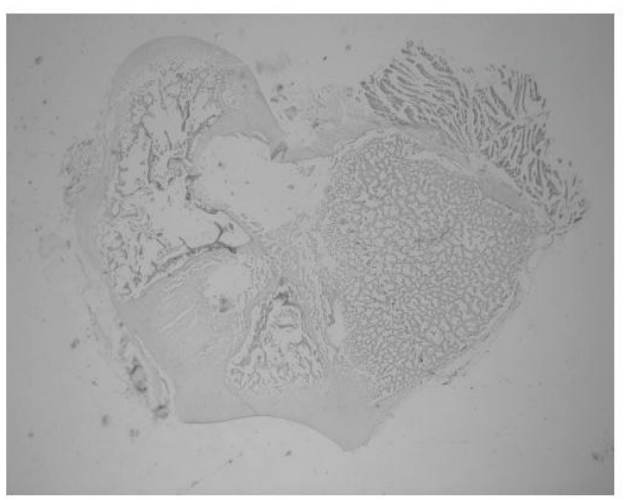

a)

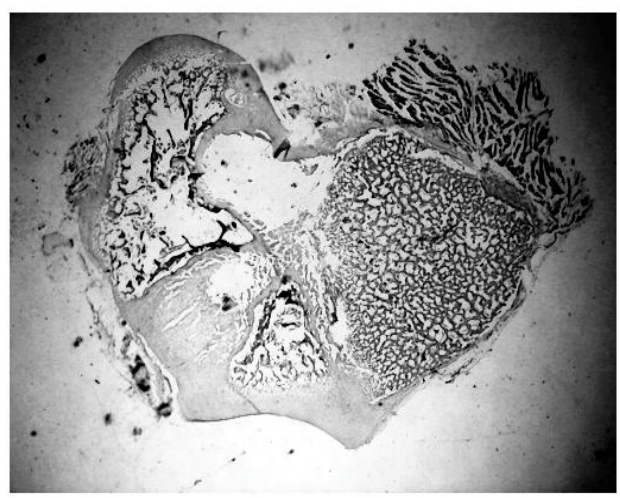

b)

Figure 1. a) Lightness image (epiphysis region); b) Equalized Lightness image 


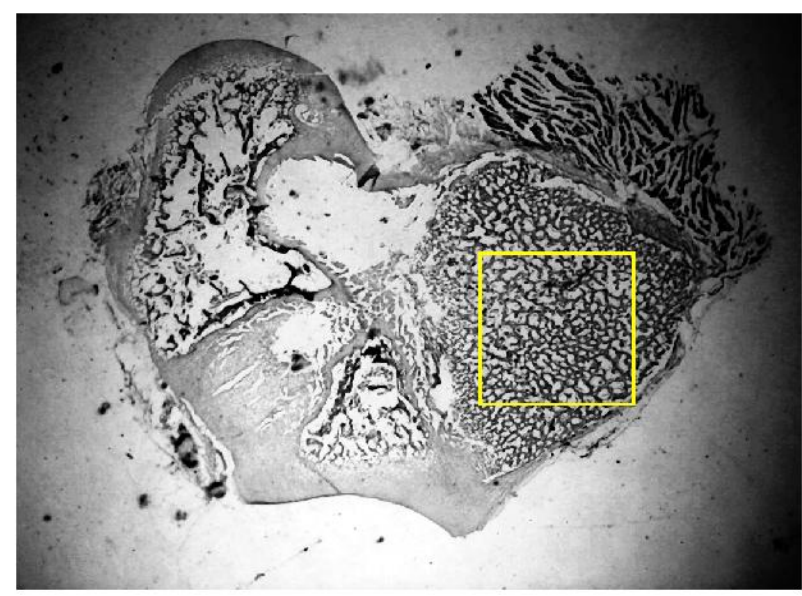

a)

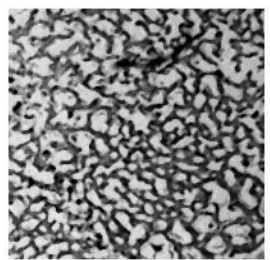

b)

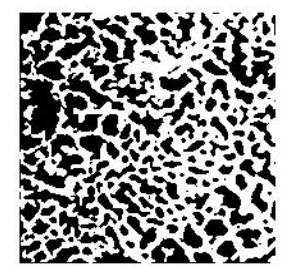

c)

Figure 2. a) Selection of region of interest (ROI); b) ROI; c) Otsu threshold

The interest region has trabeculae which are quantified in this study. An Otsu threshold ${ }^{16}$ was applied in order to obtain a binarized image (Figure 3). This tool is an automatic method that finds an optimum threshold value to divide the image.

With the last process, it remains some undesired particles (noise); these structures are removed by using an opening by reconstruction filter (Equation 2). This filter eliminates undesired objects in the image without modify the shape of the interest regions ${ }^{17,18,19}$.

$$
\tilde{\gamma}_{\lambda}(f)=\lim _{n \rightarrow \alpha} \delta_{f}^{n}\left(\varepsilon_{\lambda}(f)\right)=\delta_{f}^{1} \delta_{f}^{1} \cdots \delta_{f}^{1}\left(\varepsilon_{\lambda}(f)\right)
$$

The manual measurement of trabeculae is performed by using a metric reference in the trabecula center. Taking account of this consideration, one way to measure this component is setting a line in the center of each trabecula; for this reason the skeleton of the image was employed (Figure 3). The skeleton is a group of lines with one pixel width that preserve the topology and shape of the object. 


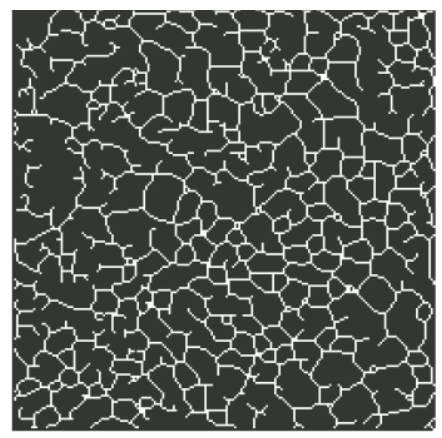

Figure 3. Image skeleton

There are different ways to get the skeleton of one image, all of them reported in different researches ${ }^{20}$. One of them is the homotopic sequential thinning $^{21}$, that consist in an image thinning applying structural homotopic elements in sequence (in this case 8) and its rotation until idempotence, preserving the homotopy of the figure (Equation 3). A sequential thinning of one image $\mathrm{X}$ by a structural element $\mathrm{B}$ and its $\mathrm{n}$ possible discrete rotations is denoted by $X \underline{\mathrm{O} B}$.

$$
X \underline{\mathrm{O}} B=\left(\cdots\left(\left(X \mathrm{O} \Theta_{1} B\right) \mathrm{O} \Theta_{2} B\right) \mathrm{O} \cdots\right) \mathrm{O}_{8}{ }_{8} B
$$

\subsection{Experimentation}

The skeleton has branches, and each element in the branch is considered one trabecula; these little trabecular structures were separated through the algorithm developed in $\mathrm{C}++$; the process follows the lines in the skeleton taking account the neighbors, of pixels. Each pixel of the trabecula is stored in a FIFO (First In, First Out) auxiliary structure while the region is explored. A FIFO is a method of data structuring that is widely used by computer programmer and it is implemented with pointers.

The algorithm starts with the searching of the pixels in the image; when one pixel of the skeleton is detected, a new process is performed by checking the neighbors that below to the line of the skeleton. In order to find the neighbors and their angles, a $3 \times 3$ matrix is followed labeling the neighbors and their corresponding angles that are in the skeleton. Once the coordinates and the angles of the neighbors are stored, four cases can be found: 1) There are no neighbors; 2) There is one neighbor; 3) There are two neighbors; and 4) There are three neighbors. If there is no neighbor, the pixel is considered an isolated point and it is not taken into account. If one neighbor is found, each pixel is stored into the auxiliary FIFO that is used to following the skeleton. In case none connection is found at the end of the 
line, the algorithm verifies if this pixel is the end of the line. In an affirmative case, longitude and the coordinates of the starting and ending points are stored in other FIFO structure; this new structure (FIFO2) is specially used for trabeculae register. If two neighbors were found, it is necessary to consider the others neighborhood (Figure 4), because the line can be a zigzag line or the middle of one trabecula.

\begin{tabular}{|c|c|c|c|c|c|c|c|}
\hline \begin{tabular}{|l|l|l|}
0 & 1 & 1 \\
$P$ & 1 & 1 \\
0 & 1 & 0 \\
\end{tabular} & \begin{tabular}{|l|l|l|}
0 & 1 & 0 \\
$P$ & 1 & 1 \\
0 & 1 & 1 \\
\end{tabular} & \begin{tabular}{|l|l|l|}
0 & 1 & 0 \\
1 & 1 & $P$ \\
1 & 1 & 0 \\
\end{tabular} & \begin{tabular}{|l|l|l|}
1 & 1 & 0 \\
1 & 1 & $P$ \\
0 & 1 & 0 \\
\end{tabular} & \begin{tabular}{|l|l|l|}
0 & $P$ & 0 \\
1 & 1 & 1 \\
0 & 1 & 1 \\
\end{tabular} & \begin{tabular}{|l|l|l|}
0 & $P$ & 0 \\
1 & 1 & 1 \\
1 & 1 & 0 \\
\end{tabular} & \begin{tabular}{|l|l|l|}
0 & 1 & 1 \\
1 & 1 & 1 \\
0 & $\mathrm{P}$ & 0 \\
\end{tabular} & \begin{tabular}{|l|l|l|}
1 & 1 & 0 \\
1 & 1 & 1 \\
0 & $\mathrm{P}$ & 0 \\
\end{tabular} \\
\hline \multicolumn{8}{|c|}{ a) } \\
\hline $0 / 0$ & \begin{tabular}{l|l}
10 \\
\end{tabular} & \begin{tabular}{|l|l|l|}
1 & 0 & 0 \\
\end{tabular} & \begin{tabular}{|l|l|l|}
0 & 0 & 1 \\
\end{tabular} & \begin{tabular}{|l|l|l|}
0 & 0 & 0 \\
\end{tabular} & \begin{tabular}{|l|l|l|}
1 & 0 & 0 \\
\end{tabular} & \begin{tabular}{|l|l|l|}
0 & 0 & 1 \\
\end{tabular} & \begin{tabular}{|l|l|l|}
1 & 0 & 0 \\
\end{tabular} \\
\hline \begin{tabular}{|l|l|l|}
0 & $\mathrm{P}$ & 0 \\
\end{tabular} & \begin{tabular}{|l|l|l|}
0 & $P$ & 0 \\
\end{tabular} & \begin{tabular}{|l|l|l|}
0 & $P$ & 0 \\
\end{tabular} & 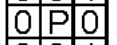 & 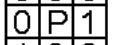 & \begin{tabular}{|l|l|l|}
0 & $\mathrm{P}$ & 1 \\
\end{tabular} & $0 \mathrm{P} 00$ & \\
\hline \begin{tabular}{|l|l|l|}
1 & 0 & 1 \\
\end{tabular} & \begin{tabular}{|l|l|l|}
0 & 0 & 0 \\
\end{tabular} & \begin{tabular}{|l|l|l|}
1 & 0 & 0 \\
\end{tabular} & \begin{tabular}{|l|l|l|}
0 & 0 & 1 \\
\end{tabular} & \begin{tabular}{|l|l|l|}
1 & 0 & 0 \\
\end{tabular} & \begin{tabular}{|l|l|l|}
0 & 0 & 0 \\
\end{tabular} & \begin{tabular}{|l|l|l|}
1 & 0 & 0 \\
\end{tabular} & \begin{tabular}{|l|l|l|}
0 & 0 & 1 \\
\end{tabular} \\
\hline
\end{tabular}

b)

Figure 4. Neighborhoods used in the line following process

For the first eight masks (Figure 4a), the letter "P" is the analyzed pixel, the numbers " 1 " in white color are the neighbors found, and the numbers " 1 " in dark color are edge pixels that determine if it is a connection node. When one of the edge pixels is detected, the algorithm marks the "P" pixel as well as the ending point of the segment, and saves the segment longitude and the starting and ending points in the FIFO2 structure. In contrast, if no one edge pixel is found, the searching process of the line continues, and there is an update to the coordinate, considering the coordinate of one of the neighbors found.

For the last eight masks (Figure $4 \mathrm{~b}$ ), the numbers "1" indicate that the algorithm begins its search in the middle of a curve or trabecula; a flag by software is activated, and this flag is used after to continue the searching process in another direction. This process starts with the coordinate of the ending point found in the other middle segment; at the end of this task, the length of the two segments are added and the starting and ending points, as well as the trabecula longitude are stored in FIFO2.

If three neighbors is found, the pixel is considered as a connection node, and the algorithm breaks the joint by removing the neighbors found in this point, in order to separate the segment from the other; again, longitude and the starting and ending points are stored in the FIFO2 structure. When the algorithm has finished the searching process in the skeleton, the data of the FIFO2 structure is saved in a text file. Each pixel found after the starting point is part of one trabecula, this way it has counted the length of the 
trabecula. When the connection node is found, a variable is incremented; at the end, this variable shows the trabecular node distribution in the image.

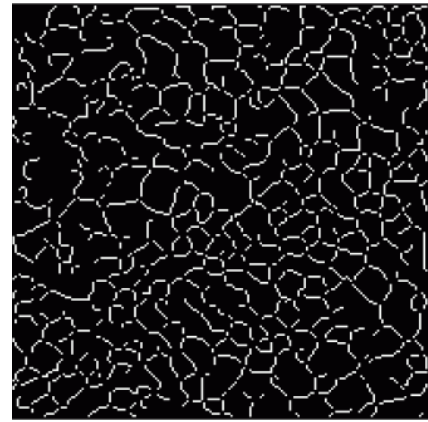

Figure 5. Segmented image

During the searching and labeling process, it is possible to find some little segment that the skeletonization process cannot eliminate. For this study case, and considering the chosen resolution $(1024 \times 768)$ for the images, the algorithm can interpret these structures as trabeculae. For this reason, in the algorithm was added an additional stage in order to evaluate if the size of the segment found can be considered as a trabecula; in this case, the minimum length established was three pixels, therefore the segments with a length less than three pixels are removed automatically. Figure 5 shows the result of this last stage.

The counting of trabeculae and connection nodes made in this study has showed important results, for example, when the procedure was applied to the last image (Figure 5), 475 connections nodes and 518 trabeculae were found; the average length is $0.06157011 \mathrm{~mm}$. Actually, the algorithm is being used with a wide selection of samples in a global study with a group test, in order to prove its effectiveness and precision with statistical methods and to compare with other methods.

The main differences with respect to the described methodologies are the following: 1) The type of images used to perform the procedure (micro-slides of bone using optical microscope); 2) proposed methodology offers an exhaustive tracking of the skeleton (to pixel-level) in order to obtain the measurement of quantity and length of the trabeculae, as well as the quantity of connections nodes (it is not an approach based on a mathematical methodology); 3) a non-commercial alternative is offered with an option of being applied to images of other types. 


\section{CONCLUSIONS}

With the application of the algorithm to the test images, an estimation of the number of trabeculae in the samples was obtained. The advantage of the proposed algorithm in comparison with commercial software (like the Bone Explora $($ ) is that the count of trabeculae is more exact, because it does not use a mesh in order to count trabeculae as the fractal method. The proposed algorithm follows line by line the skeleton until reaching a union point; this procedure permits to count exactly the quantity of trabeculae in the ROI section. The quantity of trabeculae is usually greater than connection nodes because a connection node can be shared with more trabeculae. This study is focused in the method to count trabeculae. For future approximation of trabecular distribution used as indicator of risk fracture, it is necessary to have samples of the same region, like femur neck for example; in addition, it is necessary for a larger number of samples and a depth study of the trabecula thickness in original samples (before the skeletonization process can be applied). Actually new samples are in preparation in order to do the study of trabeculae in function of age and osseous density in rats which will tray in futures studies.

Another improvement opportunity in the approach can be noted in the skeletonization process, because it can leave some residual particles that are not trabeculae, but it could be improved with a different criterion to remove those details avoiding this process in the trabeculae count algorithm. In future works, this optimization can be adding in an additional stage in the skeletonization procedure in order to remove these pixels that can be confused with trabeculae. The criterion mentioned will be determined by the length of the remnant segment, and depending of the final resolution of the image that consider the minimum size that can be selected in a slide bar.

\section{ACKNOWLEDGMENTS}

This research project has been supported by the government agency CONACyT (14059) and the "Fondo Sectorial de Investigación para la Educación" (SEP-CONACyT 2007 - México).

\section{REFERENCES}

[1] International Osteoporosis Foundation IOF, Osteoporosis and you. Buenos Aires Argentina. Retrieved on August 1, 2011, from http://www.iofbonehealth.org/.

[2] F.R. Spivacow, and A. Sánchez, Epidemiology, costs, and burden of osteoporosis in Argentina. Archives of osteoporosis, 5(1-2), p1-6, 2010. http://dx.doi.org/10.1007/s11657-010-0038-4.

[3] M.L. Bouxsein, Bone quality: Where do we go from here? 
Osteoporosis. International, 14(5), p118-127, 2003. http://dx.doi.org/10.1007/s00198-003-1489-x.

[4] D. Felsenberg, and S. Boonen, The bone quality framework: Determinants of bone strength and their interrelationships, and implications for osteoporosis management. Clinical Therapeutics, 27(1), p1-11, 2005. http://dx.doi.org/10.1016/j.clinthera.2004.12.020.

[5] J.A. Skipper, and T.N. Hangartner, Optimizing X-ray spectra for dual-energy radiographic bone densitometry. BioMedical Imaging Laboratory, 29, p297-300, 2002.

[6] V. Lozada, and O. Alberto, Comportamiento de la densitometría mineral ósea central en pacientes con factores de riesgo y fractura de cadera para el diagnostico de la osteoposoris. Revista de Sanidad Militar, 60(4), p265-269, 2006.

[7] P.K. Saha, and F.W. Wehrli, Measurement of trabecular bone thickness in the limited resolution regime of in vivo MRI by fuzzy distance transform. Transactions on Medical Imaging, 23(1), p53-62, 2004. http://dx.doi.org/10.1109/TMI.2003.819925.

[8] P.C. Watanabe, S.A. Monteiro, J.C. Campbell, and D.A. Elejalde, Análisis del patrón óseo trabecular de mandíbulas maceradas en radiografías panorámicas digitales. International Journal of Morphology, 24(3), p369-376, 2006. http://dx.doi.org/10.4067/S0717-95022006000400013.

[9] A. Zaia, R. Eleonori, P. Maponi, R. Rossi, and R. Murri, MR imaging and osteoporosis: Fractal lacunarity analysis of trabecular bone. IEEE Transactions on Information Technology Biomedicine, 10(3), p484-489, 2006. http://dx.doi.org/10.1109/TITB.2006.872078.

[10] K. Huang, D. Ta, W. Wang, and L.H. Le, Simplified inverse filter tracking algorithm for estimating the mean trabecular bone spacing. IEEE Transactions on Ultrason Ferroelectr Freq Control, 55(7), p1453-1464, 2008.

[11] J.S. Gregory, R.M. Junold, P.E. Undrill, and R.M. Aspen, Analysis of trabecular bone structure using fourier transforms and neural networks. IEEE Transactions on Information Technology in Biomedicine, 3(4), p289-294, 1999. http://dx.doi.org/10.1109/4233.809173.

[12] J.I. Pastore, E. Moler, and G. Meschino, Segmentación de biopsias de médula ósea mediante filtros morfológicos y rotulación de regiones homogéneas. Brazilian Journal of Biomedical Engineering, 21(1), p37-44, 2005.

[13] H. Blain, P. Chavassieux, N. Portero-Muzy, F. Bonnel, F. Canovas, M. Chammas, P. Maury, and P.D. Delmas, Cortical and trabecular bone distribution in the femoral neck in osteoporosis and osteoarthritis. Bone, 43(5), p862-868, 2008. http://dx.doi.org/10.1016/j.bone.2008.07.236. 
[14] A. Hanbury, Constructing cylindrical coordinate colour spaces. Pattern Recognition Letters, 29(4), p494-500, 2008. http://dx.doi.org/10.1016/j.patrec.2007.11.002.

[15] W.K. Pratt, Digital Image Processing. $\left(2^{\text {nd }} e d\right)$. New York: John Wiley \& Sons Inc., 1991.

[16] R. González, and R. Woods, Digital image processing ( $3^{\text {rd }}$ ed.). New Jersey, USA: Prentice Hall, 2008. http://dx.doi.org/10.1117/1.3115362.

[17] J. Crespo, and V. Maojo, New results on the theory of morphological filters by reconstruction. Pattern Recognition, 31(4), p419-429, 1998. http://dx.doi.org/10.1016/S0031-3203(97)00062-9.

[18] I.R. Terol, and D. Vargas, Openings and closings by reconstruction using propagation criteria. Computer Analysis of Images and Patterns, 21(24), p502-509, 2001.

[19] I.R. Terol, and D. Vargas-Vazquez, Openings and closings with reconstruction criteria: A study of a class of lower and upper leveling. Journal of Electronic Imaging, 14(1), p1-11, 2005.

[20] L. Lam, S.W. Lee, and C. Suen, Thinning methodologies: a comprehensive survey. IEEE Transactions on Patterns Analysis and Machine Intelligence, 14(9), p869-885, 1992. http://dx.doi.org/10.1109/34.161346.

[21] P. Soille, Morphological image analisis. Principles and applications ( $\left.2^{\text {nd }} e d.\right)$. New York, USA: Springer, 2003. 